4-10-2015

\title{
SDSS J14584479+3720215: A Benchmark JHKs Blazar Light Curve from the 2MASS Calibration Scans
}

James R. A. Davenport

Western Washington University, james.davenport@wwu.edu

John J. Ruan

Andrew C. Becker

Chelsea L. MaCleod

Roc M. Cutri

Follow this and additional works at: https://cedar.wwu.edu/physicsastronomy_facpubs

Part of the Stars, Interstellar Medium and the Galaxy Commons

\section{Recommended Citation}

Davenport, James R. A.; Ruan, John J.; Becker, Andrew C.; MaCleod, Chelsea L.; and Cutri, Roc M., "SDSS J14584479+3720215: A Benchmark JHKs Blazar Light Curve from the 2MASS Calibration Scans" (2015). Physics \& Astronomy. 17.

https://cedar.wwu.edu/physicsastronomy_facpubs/17 


\title{
SDSS J14584479+3720215: A BENCHMARK $J H K_{S}$ BLAZAR LIGHT CURVE FROM THE 2MASS CALIBRATION SCANS
}

\author{
James R. A. Davenport ${ }^{1}$, John J. Ruan ${ }^{1}$, Andrew C. Becker ${ }^{1}$, Chelsea L. Macleod ${ }^{2}$, And Roc M. Cutri ${ }^{3}$ \\ ${ }^{1}$ Department of Astronomy, University of Washington, Box 351580, Seattle, WA 98195, USA; jrad@ astro.washington.edu \\ ${ }^{2}$ Physics Department, The United States Naval Academy, 572c Holloway Road, Annapolis, MD 21402, USA \\ ${ }^{3}$ Infrared Processing and Analysis Center, California Institute of Technology, Pasadena, CA 91125, USA \\ Received 2014 May 9; accepted 2015 February 8; published 2015 April 3
}

\begin{abstract}
Active galactic nuclei (AGNs) are well-known to exhibit flux variability across a wide range of wavelength regimes, but the precise origin of the variability at different wavelengths remains unclear. To investigate the relatively unexplored near-IR (NIR) variability of the most luminous AGNs, we conduct a search for variability using well sampled $J H K_{s}$-band light curves from the Two Micron All Sky Survey (2MASS) calibration fields. Our sample includes 27 known quasars with an average of 924 epochs of observation over three years, as well as one spectroscopically confirmed blazar (SDSS J14584479+3720215) with 1972 epochs of data. This is the bestsampled NIR photometric blazar light curve to date, and it exhibits correlated, stochastic variability that we characterize with continuous auto-regressive moving average (CARMA) models. None of the other 26 known quasars had detectable variability in the 2MASS bands above the photometric uncertainty. A blind search of the 2MASS calibration field light curves for active galactic nucleus (AGN) candidates based on fitting CARMA(1,0) models (damped-random walk) uncovered only seven candidates. All seven were young stellar objects within the $\rho$ Ophiuchus star forming region, five with previous X-ray detections. A significant $\gamma$-ray detection $(5 \sigma)$ for the known blazar using $4.5 \mathrm{yr}$ of Fermi photon data is also found. We suggest that strong NIR variability of blazars, such as seen for SDSS J14584479+3720215, can be used as an efficient method of identifying previously unidentified $\gamma$-ray blazars, with low contamination from other AGNs.
\end{abstract}

Key words: quasars: general - surveys

\section{INTRODUCTION}

The temporal flux variability from active galactic nuclei (AGNs), detectable in nearly all wavelength regimes, contains information on the underlying emission processes and source geometry that is otherwise difficult to probe (Ulrich et al. 1997). However, precise details of the physical mechanism generating the observed nuclear variability in AGNs remain unclear (Antonucci 2013). Current and future large-scale photometric time-domain surveys have motivated many recent studies of the optical broadband variability properties of various AGN subclasses using large numbers of well-sampled light curves. This has been especially useful for AGN identification and selection (Kelly et al. 2009; Kozowski et al. 2010b; Zu et al. 2013).

Beyond the optical, large-scale surveys of AGN variability have been pursued at many other wavelengths, including the radio (Thyagarajan et al. 2011), ultraviolet (Gezari et al. 2013), and $\gamma$-ray regimes (Ackermann et al. 2012). Fewer studies of AGN variability have focused on the infrared (IR), due in part to the expectation that non-variable dust and the host galaxy dominate the emission at these wavelengths (e.g., see Kishimoto et al. 2008). Explanations of AGN variability in the optical as due to localized temperature fluctuations in inhomogeneous accretion disks (Ruan et al. 2014) or global accretion rate changes (Pereyra et al. 2006) predict little flux variability from disk emission in the IR. Reprocessing of variable optical emission by a dusty torus has been suggested by Suganuma et al. (2006) as a possible cause for the near-IR (NIR) variability, although the time lags between the optical and NIR are difficult to detect. For example McHardy et al. (2007) have shown small time lags between NIR and X-ray data for the AGN 3C273, with the NIR variability leading the
X-ray by $\sim 1.5$ days, implying this NIR variability originates near the nucleus.

A study of NIR variability in the Spitzer Deep Wide-field Survey Boötes field by Kozłowski et al. (2010a) showed that although only $1.1 \%$ of objects appear to be variable in the NIR, the vast majority of the variable objects were AGNs. Small samples of AGNs monitored in both the optical and NIR have shown that while nuclear variability is prevalent, the amplitude of variations decreases toward longer wavelengths. For example, Hönig \& Kishimoto (2011) have produced realistic models of the optical to NIR variability for NGC 4151, which show a small amplitude time lag and decreasing amplitude variability as a function of wavelength. However, this is not true for blazars, whose NIR flux is dominated by non-thermal emission from a relativistic jet, rather than circumnuclear dust which is not expected to be non-variable (Cutri et al. 1985). Recent results from optical/NIR monitoring of Fermi $\gamma$-ray blazars have shown that flat-spectrum radio quasars are more variable in the NIR than in the optical (Bonning et al. 2012; Sandrinelli et al. 2013, 2014) have used seven year photometric light curves of seven blazars, spanning the optical to NIR bands $(V R I J H K)$, to find that variability amplitudes increase with wavelength for these objects.

In this study we search for NIR variability of luminous AGNs using well sampled light curves from the Two Micron All Sky Survey (2MASS) calibration data, finding one highly variable blazar (SDSS J14584479+3720215). We explore the utility of NIR variability-based selection of AGNs, including its use in identifying counterparts to Fermi $\gamma$-ray sources. We also study the nature of non-AGN contaminants with similar NIR colors and variability properties, laying groundwork for the AGN variability science possible with current and future multiepoch IR surveys. 


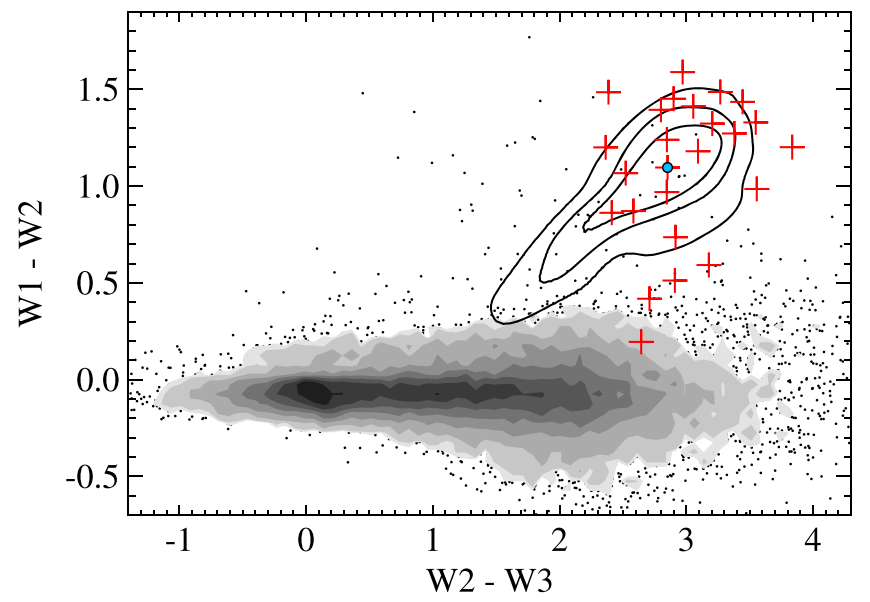

Figure 1. WISE color-color space for the Cal-PSWDB sources with WISE detections (black points and gray filled contours), known AGNs (red crosses), and the WISE blazar strip (black open contours) from Choi et al. (2014). The known blazar SDSS J14584479+3720215 is highlighted (blue circle).

\section{DATA AND AGN SELECTION}

The 2MASS survey observed the full sky in the NIR over the timespan of 1997 June to 2001 February using the $J, H$, and $K_{s}$ bands (Skrutskie et al. 2006). Photometric zero points for calibrating the survey were based on hourly observations of a set of 40 standard fields, each $8.5 \times 1^{\circ}$ in size and spaced evenly throughout the sky. These fields were visited repeatedly over the course of the survey, resulting in 562 to 3692 epochs of observation per field. This produced $J H K_{s}$ light curves for 113,030 individual objects, known as the 2MASS Calibration Point Source Working Database (hereafter Cal-PSWDB, Cutri 2006; Plavchan et al. 2008b). These light curves are the most precise NIR time domain survey to date and provide a novel data set in which to study the infrared variability properties for a wealth of astrophysical phenomena. This data set has produced the best sampled NIR light curve for an RR Lyr star (Szabó et al. 2014), a hunt for stellar flares at long wavelengths (J. R. A. Davenport et al. 2012), a large sample of young stellar objects (YSO's, Plavchan et al. 2008a; Parks et al. 2014), and many well characterized binary star systems (Becker et al. 2008; Quillen et al. 2014, J. R. A. Davenport 2015 , in preparation). Here we make use of these data to search for NIR variability of AGNs with unprecedented precision.

The Wide-field Infrared Survey Explorer (WISE, Wright et al. 2010) has created the deepest mid-IR survey of the entire sky in four photometric bands, from 3.4 to $22 \mu \mathrm{m}$. Several recent studies have demonstrated the utility of WISE colors for effectively separating AGNs from stars and unresolved galaxies. We spatially matched the Cal-PSWDB objects to the WISE all sky data release using a match radius of 1".5. This resulted in 52,148 objects with $J H K_{S}$ light curves and a detection in at least one WISE filter. The distribution of these point sources in WISE color-color space is shown in Figure 1. For comparison, we show the WISE "blazar strip," defined in Choi et al. (2014) as the Gaussian Kernel Density Estimate of the WISE colors of blazars from Massaro et al. (2009).

To find previously known AGNs in our data, we spatially matched these $52 \mathrm{~K}$ objects to the "Milliquas Catalog," version 3.3. ${ }^{4}$ We recovered 27 known AGNs with light curves in

\footnotetext{
http://quasars.org/milliquas.htm
}

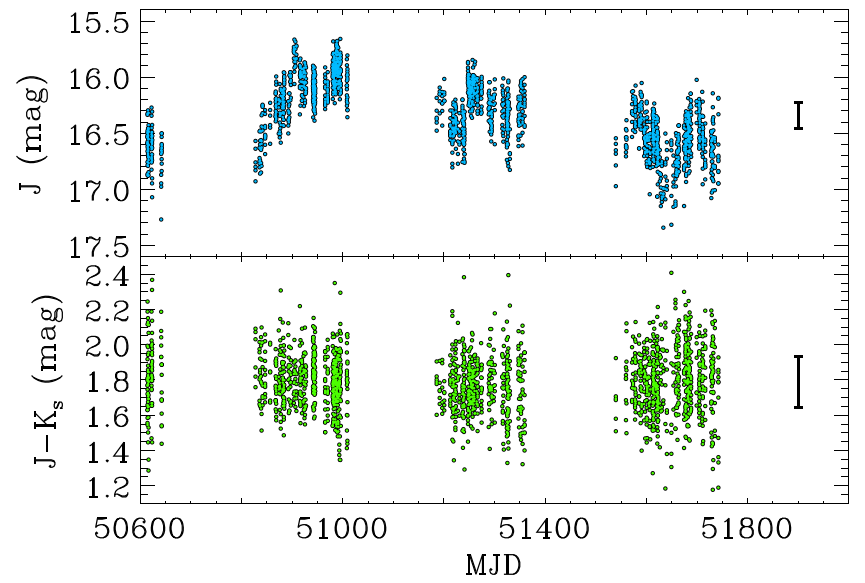

Figure 2. $J$-band (top) and $J-K_{S}$ color (bottom) light curves for the known blazar, SDSS J14584479+3720215. The median photometric error is shown for comparison (vertical bar).

Cal-PSWDB, 25 of which had WISE colors shown in Figure 1. These AGNs had between 26 and 3482 epochs of Cal-PSWDB photometry, with an average of 924 epochs. Of these, 19 have been spectroscopically confirmed by the Sloan Digital Sky Survey (SDSS; York et al. 2000; Bovy et al. 2011; Pâris et al. 2012), one of which has been identified as a blazar (SDSS $\mathrm{J} 14584479+3720215)$. We have listed the 2MASS object identifications and photometric properties of the 27 matched "Milliquas" AGNs in Table 1.

\section{AGN VARIABILITY IN THE NIR}

This set of known AGNs with well sampled Cal-PSWDB light curves creates a unique data set in which to search for AGN variability in the NIR. To determine if these objects were variable in the 2MASS bands, we computed the error-weighted root mean square variability for each light curve, $\Sigma$, following the prescription in Sesar et al. (2007). Objects were classified as variable if they had $\Sigma_{J}>10^{-2}$ mag. Of the "Milliquas" AGNs in our data set, only the known blazar, SDSS J14584479 +3720215 , exhibited variability above this threshold. The $J$ band and $J-K_{s}$ color light curves for this blazar, with 1972 epochs of Cal-PSWDB photometry spanning over $3 \mathrm{yr}$, are presented in Figure 2. The remaining 26 known AGNs in our sample displayed no significant NIR variability using this metric.

We found strong variability in SDSS J14584479+3720215 in all three 2MASS bands with our densely sampled light curves. However, as seen in Figure 2 no significant variation in $J-K_{s}$ color was found for this object, indicating the underlying variability was "gray" over this wavelength regime. A larger sample of such well studied variable objects is needed to test if this is truly a generic property of blazars in the NIR.

Characterizing photometric variability timescales has proven to be a very efficient means of selecting a clean sample of AGNs from time domain surveys (e.g., Kozowski et al. 2010b; Butler \& Bloom 2011; MacLeod et al. 2011). The so-called "damped random walk" (DRW) model provides a robust fit to AGN light curves as a function of two observable free parameters: the characteristic timescale $\tau$, and the variability amplitude $\mathrm{SF}_{\infty}$. Stars are easily removed when fit with this model, as their primary sources of variability are typically stochastic (e.g., flares or flickering) or strongly periodic (such 

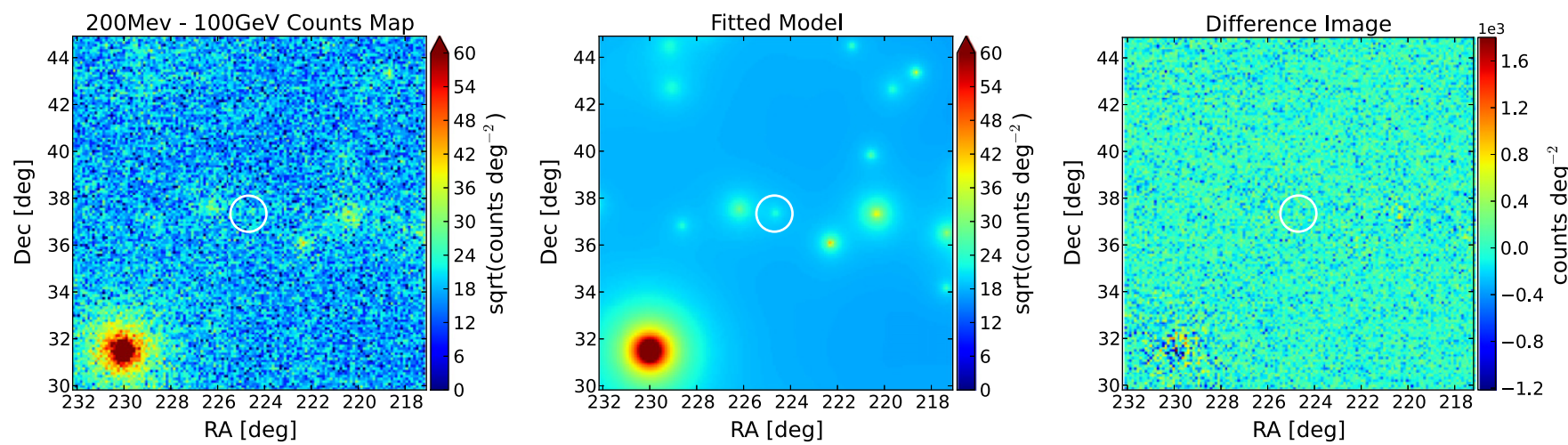

Figure 3. Left: $200 \mathrm{MeV}-100 \mathrm{Gev}$ counts maps of $\sim 4.5 \mathrm{yr}$ of Fermi data, centered on the known blazar (circled). Middle: simulated counts map for the best fit model of all sources in the field, including the known blazar, detected with TS $=30$. Right: difference image between the observed and model counts maps.

Table 1

NIR Properties of the 27 "Milliquas" AGNs Found in the Cal-PSWDB

\begin{tabular}{|c|c|c|c|c|c|c|}
\hline 2MASS ObjectID & $N_{\text {epoch }}(J)$ & $\langle J\rangle$ & $\left\langle J-K_{s}\right\rangle$ & $W 1$ & $W 1-W 2$ & $W 2-W 3$ \\
\hline J002410.86-015646.6 & 1063 & 16.86 & 1.45 & 14.91 & 1.33 & 3.55 \\
\hline J015454.88+004044.6 & 30 & 16.96 & 1.43 & 15.80 & 1.41 & 3.05 \\
\hline J015451.41+005933.2 & 339 & 17.00 & 1.51 & 15.29 & 0.42 & 2.71 \\
\hline J015429.74+002711.0 & 86 & 17.03 & 1.52 & 15.61 & 1.27 & 3.38 \\
\hline J034105.84+070917.4 & 11 & 17.08 & 1.18 & 15.39 & 1.48 & 2.39 \\
\hline J055708.14-002414.3 & 254 & 16.99 & 1.48 & $\ldots$ & $\ldots$ & $\ldots$ \\
\hline J085116.85+120028.6 & 2580 & 16.72 & 1.75 & 13.58 & 0.97 & 2.85 \\
\hline J094235.96+591354.7 & 68 & 17.08 & 1.49 & 15.22 & 1.49 & 3.27 \\
\hline J094249.43+593206.6 & 778 & 16.23 & 1.56 & 13.39 & 0.87 & 2.58 \\
\hline J122125.95-001006.1 & 1873 & 16.68 & 1.41 & 14.89 & 0.51 & 2.91 \\
\hline $\mathrm{J} 122131.10+000742.7$ & 9 & 17.01 & $\ldots$ & 15.20 & 1.32 & 3.20 \\
\hline J122144.68-001141.5 & 19 & 17.09 & 1.76 & 15.78 & 1.43 & 3.44 \\
\hline $\mathrm{J} 122152.13+001719.0$ & 49 & 17.02 & 1.41 & 15.77 & 0.59 & 3.18 \\
\hline $\mathrm{J} 121427.03+350907.9$ & 2737 & 16.29 & 1.10 & 13.38 & 1.24 & 2.85 \\
\hline J121418.95+352920.6 & 18 & 17.10 & $\ldots$ & 16.50 & 1.20 & 3.84 \\
\hline $\mathrm{J} 121408.54+355021.5$ & 1212 & 16.91 & 1.56 & 14.63 & 0.20 & 2.64 \\
\hline J144057.24-000951.1 & 916 & 16.40 & 1.13 & 13.80 & 1.59 & 2.97 \\
\hline $\mathrm{J} 150026.22-005428.2$ & 1568 & 16.79 & 1.64 & 14.16 & 1.18 & 3.09 \\
\hline J150043.39-005820.7 & 1500 & 16.50 & 1.48 & 13.88 & 0.74 & 2.92 \\
\hline $\mathrm{J} 145846.09+371235.9$ & 58 & 17.06 & 1.38 & 15.32 & 0.98 & 3.56 \\
\hline $\mathrm{J} 145844.84+372021.8^{\dagger}$ & 1927 & 16.33 & 1.77 & 14.03 & 1.10 & 2.85 \\
\hline J163124.44+295301.8 & 1191 & 15.51 & 1.73 & 12.46 & 1.07 & 2.52 \\
\hline J204110.28-052626.3 & 21 & 16.99 & 1.49 & $\ldots$ & $\ldots$ & $\ldots$ \\
\hline $\mathrm{J} 220037.69+211051.9$ & 888 & 16.75 & 1.32 & 14.34 & 1.45 & 2.90 \\
\hline J220028.41+203902.1 & 15 & 16.88 & $\ldots$ & 15.22 & 1.20 & 2.36 \\
\hline $\mathrm{J} 231808.32+001152.3$ & 7 & 16.99 & 1.46 & 15.06 & 1.39 & 2.80 \\
\hline $\mathrm{J} 231821.13+002937.2$ & 358 & 16.95 & 1.52 & 14.62 & 0.86 & 2.41 \\
\hline
\end{tabular}

Note. Our benchmark blazar is indicated $(\dagger)$.

as pulsations or rotation) in nature. Combining DRW variability models with multi-wavelength photometry provides even greater power in separating blazars from "normal" AGNs (Ruan et al. 2012).

From our Cal-PSWDB-WISE matched sample we selected all light curves having at least 50 good $J$-band epochs $(\mathrm{PH}$ _ $\mathrm{UUAL}=\mathrm{A}, \mathrm{B}$ or $\mathrm{C})$, median $J$-band magnitudes brighter than $16.5 \mathrm{mag}$, and WISE colors of $W 1-W 2>0.3$ to remove main sequence stars (J. R. A. Davenport et al. 2014). We also required sources to be detected as variable using the $\Sigma_{J}>0.01$ cut described above, and removed objects known to be periodic (binaries and pulsating variables) or long period variable stars (Plavchan et al. 2008a, J. R. A. Davenport 2014, in preparation). This yielded 22 variable Cal-PSWDB targets to search for DRW timescales, which included our known blazar object, but did not select any other previously identified AGN. For completeness we also analyzed the other 26 known "Milliquas" sources for DRW variability.

For each object we down-sampled the light curves to a single data point per night, using the median of the $J$-band photometry, which reduced the light curves to an average of 144 epochs. We then ran the DRW fitting code from Kozowski et al. (2010b) and MacLeod et al. (2010) on the reduced $J$ band light curves for all 47 objects. We used the probability thresholds outlined in MacLeod et al. (2010) to determine which light curves had significant DRW variability. The known blazar had a weakly constrained DRW timescale of $\log \tau=2.0_{-1.9}^{+4.0}$ days, and a significant driving amplitude of

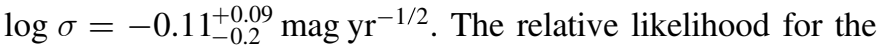
DRW versus an infinite or unconstrained timescale was $\mathrm{P}_{\text {like }}$ 
$\mathrm{P}_{\text {inf }}=1.4$, where values greater than 0.05 are considered significant. Similarly, the relative likelihood for the DRW model versus pure noise was $\mathrm{P}_{\text {like }}-\mathrm{P}_{\text {noise }}=153$, which was much greater than the threshold for significance of 0 . None of the other 26 previously identified AGNs showed any signs of significant DRW variability. However, seven of the variabilityselected objects did show significant DRW variability. We discuss the origin of these objects in the following section.

We additionally fit these light curves using the continuous auto-regressive moving average, or $\operatorname{CARMA}(p, q)$, models of Kelly et al. (2014), where $p$ is the auto-regressive order and $q$ the moving average order. The DRW is a special case of the CARMA models, equivalent to a CARMA $(1,0)$, or a first-order continuous auto-regressive process with no moving average. In the CARMA formalism, a DRW model's power spectral density is described as a single Lorentzian function with a characteristic break frequency. As mentioned above, the DRW has been shown to empirically model the behavior of QSO light curves well, but is otherwise inflexible. By looking at higher order CARMA models we search for more complex behavior in the light curve power spectral density, such as stochastic variability on multiple timescales or quasi-periodic oscillations. For SDSS J14584479+3720215 we found a moderate preference for the CARMA $(2,0)$ model compared to a DRW when using the deviance information criterion for model selection. The DRW model provides a sufficient fit to the data, in that the sequence of residuals is independently and normally distributed. A Markov Chain Monte Carlo analysis of the CARMA $(1,0)$ model provided $1 \sigma$ confidence levels on the timescale of $31<\tau<115$ days, and on the standard deviation of the driving noise of between 0.14 and 0.20 magnitudes.

\section{YSO CONTAMINATION}

The recovered DRW timescales for the seven variabilityselected objects we found in the previous section had an average timescale of $\log \tau=1.05$ days, significantly shorter than the typical timescale seen in the optical for AGNs (MacLeod et al. 2010), as well as for our blazar target. This was reproduced in the $\operatorname{CARMA}(1,0)$ models as well. These seven objects all reside in field \# 90009, which was centered on the star forming region $\rho$ Ophiuchus, making these likely YSOs. Of these YSOs, five had X-ray detections in the literature, one coming from a targeted X-ray monitoring campaign of the field (Gagné et al. 2004), and four from the Chandra X-ray source catalog (Evans et al. 2010). The other two YSOs in our sample did not have a published X-ray counterpart within 0.1 . NIR light curve properties for YSOs within this field in the Cal-PSWDB have been studied in great detail by Plavchan et al. (2008a) and Parks et al. (2014).

These seven YSOs had mid-IR colors that placed them within the blazar strip, NIR variability amplitudes comparable to our known blazar, and light curves best parameterized by a CARMA $(1,0)$ model. We thus highlight YSOs as a potentially important source of contamination for classifying AGNs from future multi-wavelength time domain searches. While using longer wavelength filters can improve the efficiency of selecting AGNs from normal stars (e.g., Massaro et al. 2012), YSOs remain a potential contaminant for object classification (see also the color spaces in Figure 7 of Koenig et al. 2012). However, these objects may be distinguished from bona fide AGNs based on their $\sim 10 \mathrm{X}$ shorter characteristic timescales under a CARMA $(1,0)$ model. YSOs are also mostly confined to the Galactic disk and dense star forming regions, and as such can be avoided by surveys.

\section{A $\gamma$-RAY DETECTION OF SDSS J14584479+3720215}

The Fermi Gamma-ray Space Telescope provides the deepest survey to date in the $100 \mathrm{MeV}$ to $100 \mathrm{GeV}$ regime, and has discovered $886 \gamma$-ray AGN in the 2nd Fermi AGN Catalog (Ackermann et al. 2011). The vast majority of these AGNs are blazars. However, $30 \%$ of Fermi sources in the Fermi 2nd Point Source Catalog (2FGL; Nolan et al. 2012) are unidentified, largely due to the poor angular resolution of Fermi's pair-conversion Large Area Telescope (LAT; Atwood et al. 2009). All reliably associated AGNs in the 2nd Fermi AGN Catalog are confidently detected by Fermi with Test Statistic TS $>25$, corresponding to approximately $>5 \sigma$ detections. For a Fermi counts map of a region of interest (ROI) centered on a new possible source, the TS detection significance is the defined to be the ratio of likelihoods TS $=2$ $(\log L($ source $)-\log L($ nosource $))$, where $L($ source $)$ is the likelihood of a model of all known sources in the ROI and a new source at the central location, while $L$ (nosource) is the likelihood for the same model but no new source at the central location (Nolan et al. 2012). The detection significance is approximately $\sqrt{\mathrm{TS}}$. Thousands of potential faint $\gamma$-ray blazars lie below the TS $>25(>5 \sigma)$ detection threshold, but are difficult to localize due to extremely low photon counts. These faint $\gamma$-ray blazars have large positional uncertainties, with error ellipses with radius $\gtrsim 20^{\prime}$ for $3 \sigma$ Fermi detections using $4 \mathrm{yr}$ of photon data (Ballet \& Burnett 2013; Thompson et al. 2014).

The strong $J H K_{s}$ variability exhibited by the blazar SDSS $\mathrm{J} 14584479+3720215$ relative to the quasars in our sample suggests that this NIR variability is likely to be indicative of strongly beamed jets, which are characteristic of blazars and are known to produce strong $\gamma$-ray emission. This allows for NIRvariability based identification of previously unidentified or undetected $\gamma$-ray blazars. SDSS J14584479+3720215 was not included in the Fermi 2FGL catalog, and thus had TS $<25$ is $2 \mathrm{yr}$ of photon data. However, motivated by the strong NIR variability we observed, we investigated whether this blazar can be detected at high significance using more photon data. We downloaded approximately $4.5 \mathrm{yr}$ of available Pass 7 Fermi photon data of the Source event class, spanning Mission Elapsed Time 239557417-383570220 s. We selected photons

in the $200 \mathrm{MeV}$ to $100 \mathrm{GeV}$ energy range, within a $10^{\circ} \mathrm{ROI}$ centered on the blazar. Figure 3 shows the raw Fermi photon counts map, along with our modeled flux map.

Using the standard binned likelihood analysis scripts provided in the Fermi Science Tools package, we performed photon event selection with an ROI-based zenith angle cut of $<100^{\circ}$ and a rock angle cut of $<52^{\circ}$. We produced a spatial model of the likely $\gamma$-ray sources within $15^{\circ}$ of the blazar based on the 2FGL catalog, using the 2FGL best fit values for the template spectral model of these sources. We included the blazar SDSS J14584479+3720215 as an additional point source, modeled as a simple power-law spectrum. The spectral normalization for all sources within $15^{\circ}$ was allowed to vary, and all spectral parameters were set as free parameters within the ROI. We included the appropriate two year Pass 7 Galactic diffuse emission and extragalactic isotropic diffuse emission models in our source model fit to the observed counts map. The 
blazar SDSS J14584479+3720215 is detected with TS $=30$, approximately a $5 \sigma$ detection, with a $\gamma$-ray power-law spectral slope of $2.40 \pm 0.18$, typical of Fermi blazars (Ackermann et al. 2011). This detection significance is consistent with the $\mathrm{TS} \simeq 30$ found in a preliminary version of the Fermi fouryear point source catalog by Ballet \& Burnett (2013), produced using $4 \mathrm{yr}$ of photon data. We note minor discrepancies in our modeling of the brightest object in the field, noticeable in the lower-left corner of the difference image. These are wellknown issues in Fermi source analysis caused by the finite resolution of the counts maps, and do not affect our detection.

\section{DISCUSSION}

We have conducted a search for NIR variability from AGNs using the 2MASS $\mathrm{C}$ al-PSWDB light curves. No significant variability was found for 26 of the 27 known AGNs in our sample. One previously known AGN, showed strong variations in the 2MASS filters, and we have presented the 1972 epoch NIR photometric light curve for this blazar (SDSS J14584479 +3720215 ). This previously known blazar is a benchmark object, displaying almost one magnitude of variability in the $J H K_{s}$ bands, but with no significant variation in $J-K_{s}$ color. This is the best sampled NIR light curve of a blazar ever measured. We note a handful of other AGN candidates from the 2MASS Cal-PSWDB were recently identified by Quillen et al. (2014), including the blazar SDSS J14584479+3720215.

Our detection of SDSS J14584479+3720215 as a $\gamma$-ray source, selected by infrared variability, has implications on the association and classification of multi-wavelength counterparts of Fermi sources. Current selection methods such as mid-IR color (Massaro et al. 2012) and optical variability (Ruan et al. 2012) suffer from low efficiency. This is often due to "normal" Type 1 quasars, which have similar colors in the midIR and are also variable in the optical. As this pilot study demonstrates, these issues may be surmounted by the inclusion of IR variability information, since normal AGNs are not significantly variable at this wavelength regime.

A search for other such light curves in the 2MASS CalPSWDB reveals a large number of contaminating YSOs. This study establishes a baseline for the AGN variability science possible with current and future multi-epoch IR missions such as the WISE, the Wide Field Infrared Survey Telescope (WFIRST; Green et al. 2012), and Vista Variables in the Vía Láctea (VVV; Minniti et al. 2010) surveys.

We thank P. Plavchan and J. Parks for helpful discussions of YSO variability and for sharing an early version of their manuscript. The authors acknowledge support from NASA ADP grant NNX09AC77G and NNX14AK26G. This publication makes use of data products from the Two Micron All Sky Survey, which is a joint project of the University of Massachusetts and the Infrared Processing and Analysis Center/California Institute of Technology, funded by the National Aeronautics and Space Administration and the National Science Foundation. This research has made use of the NASA/IPAC Extragalactic Database (NED) which is operated by the Jet Propulsion Laboratory, California Institute of Technology, under contract with the National Aeronautics and Space Administration. This research has made use of data obtained from the Chandra Source Catalog, provided by the
Chandra X-ray Center (CXC) as part of the Chandra Data Archive. This publication makes use of data products from the Wide-field Infrared Survey Explorer, which is a joint project of the University of California, Los Angeles, and the Jet Propulsion Laboratory/California Institute of Technology, funded by the National Aeronautics and Space Administration.

\section{REFERENCES}

Ackermann, M., Ajello, M., Allafort, A., et al. 2011, ApJ, 743, 171 Ackermann, M., Ajello, M., Allafort, A., et al. 2012, ApJ, 747, 104 Antonucci, R. 2013, Natur, 495, 165

Atwood, W. B., Abdo, A. A., Ackermann, M., et al. 2009, ApJ, 697, 1071

Ballet, J., \& Burnett, T. 2013, in Int. Cosmic Ray Conf. 33 Fermi LAT Third Source Catalog; http://www.cbfp.br/ icrc2013/papers/icrc2013-1153. pdf, 256

Becker, A. C., Agol, E., Silvestri, N. M., et al. 2008, MNRAS, 386, 416

Bonning, E., Urry, C. M., Bailyn, C., et al. 2012, ApJ, 756, 13

Bovy, J., Hennawi, J. F., Hogg, D. W., et al. 2011, ApJ, 729, 141

Butler, N. R., \& Bloom, J. S. 2011, AJ, 141, 93

Choi, Y., Gibson, R. R., Becker, A. C., et al. 2014, ApJ, 782, 37

Cutri, R. M. 2006, Explanatory Supplement to the 2MASS All Sky Data Release and Extended Mission Products, http://ipac.caltech.edu/2mass/ releases/allsky/doc/explsup.html

Cutri, R. M., Wisniewski, W. Z., Rieke, G. H., \& Lebofsky, M. J. 1985, ApJ, 296, 423

Davenport, J. R. A., Becker, A. C., Kowalski, A. F., et al. 2012, ApJ, 748, 58 Davenport, J. R. A., Ivezić, Ž., Becker, A. C., et al. 2014, MNRAS, 440, 3430 Evans, I. N., Primini, F. A., Glotfelty, K. J., et al. 2010, ApJS, 189, 37 Gagné, M., Skinner, S. L., \& Daniel, K. J. 2004, ApJ, 613, 393

Gezari, S., Martin, D. C., Forster, K., et al. 2013, ApJ, 766, 60

Green, J., Schecter, P., Baltay, C., et al. 2012, arXiv:1208.4012G

Hönig, S. F., \& Kishimoto, M. 2011, A\&A, 534, A121

Kelly, B. C., Bechtold, J., \& Siemiginowska, A. 2009, ApJ, 698, 895

Kelly, B. C., Becker, A. C., Sobolewska, M., Siemiginowska, A., \& Uttley, P. 2014, ApJ, 788, 33

Kishimoto, M., Antonucci, R., Blaes, O., et al. 2008, Natur, 454, 492 Koenig, X. P., Leisawitz, D. T., Benford, D. J., et al. 2012, ApJ, 744, 130 Kozłowski, S., Kochanek, C. S., Stern, D., et al. 2010, ApJ, 716, 530 Kozowski, S., Kochanek, C. S., Udalski, A., et al. 2010, ApJ, 708, 927 MacLeod, C. L., Brooks, K., Ivezić, Ž., et al. 2011, ApJ, 728, 26 MacLeod, C. L., Ivezić, Ž., Kochanek, C. S., et al. 2010, ApJ, 721, 1014 Massaro, E., Giommi, P., Leto, C., et al. 2009, A\&A, 495, 691 Massaro, F., D'Abrusco, R., Tosti, G., et al. 2012, ApJ, 750, 138 McHardy, I., Lawson, A., Newsam, A., et al. 2007, MNRAS, 375, 1521 Minniti, D., Lucas, P. W., Emerson, J. P., et al. 2010, NA, 15, 433 Nolan, P. L., Abdo, A.A., Ackermann, A., et al. 2012, ApJS, 199, 31 Pâris, I., Petitjean, P., Aubourg, É., et al. 2012, A\&A, 548, A66 Parks, J. R., Plavchan, P., White, R. J., \& Gee, A. H. 2014, ApJS, 211, 3 Pereyra, N. A., Vanden Berk, D. E., Turnshek, D. A., et al. 2006, ApJ, 642, 87 Plavchan, P., Gee, A. H., Stapelfeldt, K., \& Becker, A. 2008, ApJ, 684, L37 Plavchan, P., Jura, M., Kirkpatrick, J. D., Cutri, R. M., \& Gallagher, S. C. 2008, ApJS, 175, 191

Quillen, A. C., Ciocca, M., Carlin, J. L., Meng, Z., \& Bell, C. P. M. 2014, MNRAS, 441, 2691

Ruan, J. J., Anderson, S. F., Dexter, J., \& Agol, E. 2014, ApJ, 783, 105

Ruan, J. J., Anderson, S. F., MacLeod, C. L., et al. 2012, ApJ, 760, 51

Sandrinelli, A., Covino, S., \& Treves, A. 2014, A\&A, 562, A79

Sandrinelli, A., Treves, A., Falomo, R., et al. 2013, AJ, 146, 163

Sesar, B., Ivezić, Ž., Lupton, R. H., et al. 2007, AJ, 134, 2236

Skrutskie, M. F., Cutri, R. M., Stiening, R., et al. 2006, AJ, 131, 1163

Suganuma, M., Yoshii, Y., Kobayashi, Y., et al. 2006, ApJ, 639, 46

Szabó, R., Ivezić, Ž., Kiss, L. L., et al. 2014, ApJ, 780, 92

Thompson, D. J., Ballet, J., \& Burnett, T. Fermi Large Area Telescope Collaboration 2014, American Astronomical Society Meeting Abstracts, Vol. 223, 256

Thyagarajan, N., Helfand, D. J., White, R. L., \& Becker, R. H. 2011, ApJ, 742, 49

Ulrich, M.-H., Maraschi, L., \& Urry, C. M. 1997, ARA\&A, 35, 445

Wright, E. L., Eisenhardt, P.R.M., Mainzer, A. K., et al. 2010, AJ, 140, 1868 York, D. G., Adelman, J., Anderson, J.E., Jr., et al. 2000, AJ, 120, 1579

Zu, Y., Kochanek, C. S., Kozłowski, S., \& Udalski, A. 2013, ApJ, 765, 106 